\title{
QUEEN'S
UNIVERSITY
BELFAST
}

\section{Effect of Tissue Boundaries on the Intra-Body Communication Channel at $2.38 \mathrm{GHz}$}

El-Saboni, Y., Conway, G., \& Scanlon, W. (2017). Effect of Tissue Boundaries on the Intra-Body Communication Channel at $2.38 \mathrm{GHz}$. In 2017 International Workshop on Antenna Technology: Small Antennas, Innovative Structures, and Applications (iWAT) (pp. 289-292). Institute of Electrical and Electronics Engineers Inc.. https://doi.org/10.1109/IWAT.2017.7915381

Published in:

2017 International Workshop on Antenna Technology: Small Antennas, Innovative Structures, and Applications (iWAT)

\section{Document Version:}

Peer reviewed version

Queen's University Belfast - Research Portal:

Link to publication record in Queen's University Belfast Research Portal

Publisher rights

(C) 2017 IEEE. This work is made available online in accordance with the publisher's policies. Please refer to any applicable terms of use of the publisher

\section{General rights}

Copyright for the publications made accessible via the Queen's University Belfast Research Portal is retained by the author(s) and / or other copyright owners and it is a condition of accessing these publications that users recognise and abide by the legal requirements associated with these rights.

Take down policy

The Research Portal is Queen's institutional repository that provides access to Queen's research output. Every effort has been made to ensure that content in the Research Portal does not infringe any person's rights, or applicable UK laws. If you discover content in the Research Portal that you believe breaches copyright or violates any law, please contact openaccess@qub.ac.uk. 


\title{
Effect of Tissue Boundaries on The Intra-Body Communication Channel at $2.38 \mathrm{GHz}$
}

\author{
Yomna El-Saboni, Gareth A. Conway, William G. Scanlon \\ Centre for Wireless Innovation, Institute of Electronics, Communication \& Information Technology \\ Queen's University Belfast, BT3 9DT, UK \\ yelsaboni01@qub.ac.uk, g.conway@qub.ac.uk,w.scanlon@qub.ac.uk
}

\begin{abstract}
A study of the intra-body propagation channel between two identical tissue implanted antennas is presented. To investigate the effect of the tissue boundaries, the channel between the two implants is evaluated within a tissue layered numerical phantom with both insulated and un-insulated antenna structures in the MedRadio operating band $(2.36-2.40 \mathrm{GHz})$. The results demonstrate how wave propagation between the antennas inside the same block of tissue is largely unaffected by changes in the peripheral surrounding tissues, irrespective of their material characteristics. On the contrary, propagation across tissue boundaries is affected by the boundary and the path distance within each tissue according to the dielectric parameters involved.
\end{abstract}

Keywords-Intra-body; implantable antennas; hetergenous human tissue phantom; path gain; medical device.

\section{INTRODUCTION}

An Intra-Body Network (IBN) involves the co-ordination of miniaturized implanted wireless medical devices operating within the human body. The implementation of IBNs enables faster and more efficient smart prosthetics that can restore movement for paralyzed patients and can help provide control over many vital body systems. The majority of previous research in the field of body area networks involves external channels to the body [1]. However, networking implantable devices is more challenging as the signals traverse the lossy dispersive body tissues and organs [2]. Therefore, despite their importance to the medical industry, there have been fewer and less detailed studies published for IBNs in comparison with the work done in other body area networks. Consequently, there is a need to investigate and better understand propagation between two or more implant antennas within the same host body. In this paper we utilize numerical modeling of the link between two identical antennas embedded within a multi-layered cylindrical tissue equivalent phantom and investigate the effect of tissue boundaries on the channel gain.

\section{INTRA-BODY ANTENNAS AND PROPAGATION}

The human body is a complex structure with many tissue interfaces and layers. A propagating wave that traverses tissue boundaries will suffer reflection, refraction and transmission losses according to several factors such as the different dielectric properties of the tissues, the boundary shape and the angle of wave incidence, all of which dramatically affect the path loss between implant antennas [2]. Wave absorption in high water content tissues can be quite significant, particularly for deep tissue implants [3]. Additionally, the nature of the tissues surrounding an implant antenna can affect its performance and its resonating frequency. Different tissues exhibit different dielectric properties and this may mean that antennas need to be modified or re-tuned each time the device's position changes with respect to the surrounding tissues. Of course, this challenge is somewhat mitigated by using multiresonance antennas [4] and to some degree by the required biocompatible insulating layer for the antenna. Insulation improves radiation efficiency as near field losses are reduced and it prevents the short circuit effect associated with highly conductive tissue materials such as skin and muscle [2],[5]. Insulated antennas also have the advantage of being less sensitive to the dielectric properties of the surrounding medium [3] in terms of their current distribution and hence feed-point impedance. As well as being biocompatible, insulating materials for implant antennas should be low loss and one proposed material is polytetrafluorethylene with relative permittivity of 2.07 and conductivity of $0.25 \mathrm{~S} / \mathrm{m}$ [3].

\section{NUMERICAL MODELING}

A multi-layered cylindrical phantom model, consisting of three body tissues muscle, fat and skin, each with their specific dielectric properties, was used as the basis of the numerical modeling study. The simulations were performed in CST Microwave Studio ${ }^{\circledR}$ in three separate stages. In the first stage, three homogeneous tissue phantoms were created and the radiating field generated by an un-insulated bowtie dipole antenna considered. The bowtie dipole was designed so that it was matched in all three tissue types. In the second stage, the reflection coefficients and forward gain values were recorded for two identical un-insulated bowtie dipole antennas at various separation distances inside a multi-layered phantom. The use of the multi-tissue matched bowtie antenna ensured that the path gain results were not affected by changes in the physical volume of the antennas since in high permittivity materials such as muscle a resonant un-insulated antenna would be physically smaller and hence would be subject to higher propagation losses. In the third stage, the effect of antenna insulation is considered. Firstly, a cylindrical dipole was optimized to resonate in free space and then, by performing a numerical study within homogenous tissue phantoms, the thickness of vacuum insulation needed to preserve the same physical size of the antenna was determined. The same insulation thickness was used for the bowtie antenna, which was then re-optimized so that it was resonant in all three tissues.

\section{A. Numerical Tissue Phantom}

The human body consists of different tissue types that possess a significant heterogeneity and variation in electrical 
characteristics such as its conductivity, power absorption, path loss and permittivity [6]. Propagation between implant antennas is directly affected by the tissues which the signal passes through, as the power absorption (and hence path loss) are dependent on the thickness of each tissue and the presence of their boundaries. Therefore, a multi-layered cylindrical numerical phantom was designed with three tissue layers (muscle core, concentric fat mid layer and skin outer layer) similar to phantoms presented in [7]. The total diameter and height of the cylindrical phantom are always set at $400 \mathrm{~mm}$ but the thickness of each layer varies from one scenario to the other.

\section{B. Frequency Band}

Lower frequency bands restrict the available signal bandwidth while higher bands suffer from increased tissue absorption [8]. A relatively wide channel bandwidth is required to accommodate the future development of intelligent sensing systems and real time responsive medical systems and this has led to increased interest in higher frequency bands for implant applications. Higher frequency operation also allows the use of physically smaller antennas which is important for implantable applications. Therefore, the MedRadio Band (2.36-2.4 GHz) [9] was chosen for this study.

\section{Antenna Design}

The un-insulated broadband bowtie dipole antenna was chosen for the first stage and second stage of the study. Having a broader resonant bandwidth allows the antenna to exhibit the same physical size and resonant property when placed in the three different layers even when subject to loading by the medium's differing effective permittivity and wavelength [10]. This approach is needed for the second stage as one of the antennas changes position through the different tissue layers. The bowtie was designed by modeling it in the center of homogenous phantoms, one with each tissue type (muscle, fat and skin) and optimizing the dimensions accordingly to ensure that it was properly matched across the MedRadio band. This was achieved when the total height $(h)$ and width $(w)$ of the antenna was $60 \mathrm{~mm}$ and $20 \mathrm{~mm}$, respectively, with a delta gap source $\partial=0.2 \mathrm{~mm}$. The thickness $(k)$ of the perfect electric conductor (PEC) antenna was $0.2 \mathrm{~mm}$ and the physical design is similar to insulated version illustrated in Fig.1. Table 1 presents the achieved $\left|S_{11}\right|$ for the un-insulated bowtie dipole antenna in each of the homogenous tissue phantoms. The tissue parameters are given in the table. The last stage of the analysis required insulated antennas. A cylindrical dipole antenna was first designed for free-space resonance in the MedRadio band with a radius of $0.1 \mathrm{~mm}$ and a total length of $55 \mathrm{~mm}$. This was then modeled with different thicknesses of vacuum insulation to determine the optimum thickness at which the antenna's physical size remains constant in all tissues while still being matched across the MedRadio band. The resultant insulation radius $(t)$ was found to be $1.8 \mathrm{~mm}$ (Fig. 1). This thickness of vacuum insulation was then also applied to the bowtie dipole antennas.

\section{Simulation Scenarios}

The implant to implant link study involves three main multilayer phantom scenarios. In the first scenario, the phantom has a muscle core with a radius of $140 \mathrm{~mm}, 40 \mathrm{~mm}$ additional radius of fat tissue $(140 \mathrm{~mm}-180 \mathrm{~mm})$ and $20 \mathrm{~mm}$ additional radius of skin tissue $(180 \mathrm{~mm}-200 \mathrm{~mm})$. The phantom design is not intended to be anatomically realistic. One antenna was positioned at the center of the phantom and the second antenna is positioned along a radial line starting at $10 \mathrm{~mm}$ separation and then incremented at $3 \mathrm{~mm}$ intervals. The top and bottom edges of the antenna are perpendicular as illustrated in Fig. 2. In the second scenario as shown in Fig. 2, both antennas were held at a fixed position inside the muscle layer; one at the phantom center and the second $90 \mathrm{~mm}$ away. However, this time the fat layer increases in thickness from $10 \mathrm{~mm}$ to $90 \mathrm{~mm}$ and the muscle decreases with the same interval so that the overall size of the phantom remains the same. This is achieved by moving the muscle/fat boundary from $100 \mathrm{~mm}$ radius to $180 \mathrm{~mm}$ with $10 \mathrm{~mm}$ intervals each time. The last scenario follows the previous one, except the second antenna is placed at $185 \mathrm{~mm}$ away from the first. Hence it stays inside the fat layer throughout the study. This provides insight about the effect of the boundary when the antenna is placed in different positions. In the third stage, the first scenario was repeated with the insulated cylindrical dipole and bowtie antennas and compared with the un-insulated bowtie dipole case.

\section{RESUlTS}

Stage 1: The total electric field was evaluated for the uninsulated bowtie antennas inside the different tissue types (Fig. 3 ). This was compared with free space to highlight the effect of tissue dielectric properties on the radiating field. As expected the attenuation rate is less in the fat only phantom in comparison with all the other phantoms. While the muscle and skin have close attenuation rates which dominates the rate at which the electric field decays in a multi-layer phantom, the attenuation rate is also reduced when the electric field passes through the fat layer.

\begin{tabular}{|c|c|c|c|c|c|}
\hline \multicolumn{3}{|r|}{ TABLE 1} & \multirow{2}{*}{\multicolumn{3}{|c|}{ Frequency $(\mathrm{GHz})$}} \\
\hline Tissue & Relative & Conductivity & & & \\
\hline Tуре & Permittivity $\left(\varepsilon_{r}\right)$ & $(\sigma)(\mathbf{S} / \mathbf{m})$ & & 2.36 & 2.40 \\
\hline Muscle & 52.8 & 1.69 & & -10.42 & -10.32 \\
\hline Fat & 5.29 & 0.10 & $\begin{array}{l}\left|S_{11}\right| \\
\text { (dB) }\end{array}$ & -14.16 & -14.77 \\
\hline Skin & 38.1 & 1.43 & & -11.46 & -11.47 \\
\hline
\end{tabular}
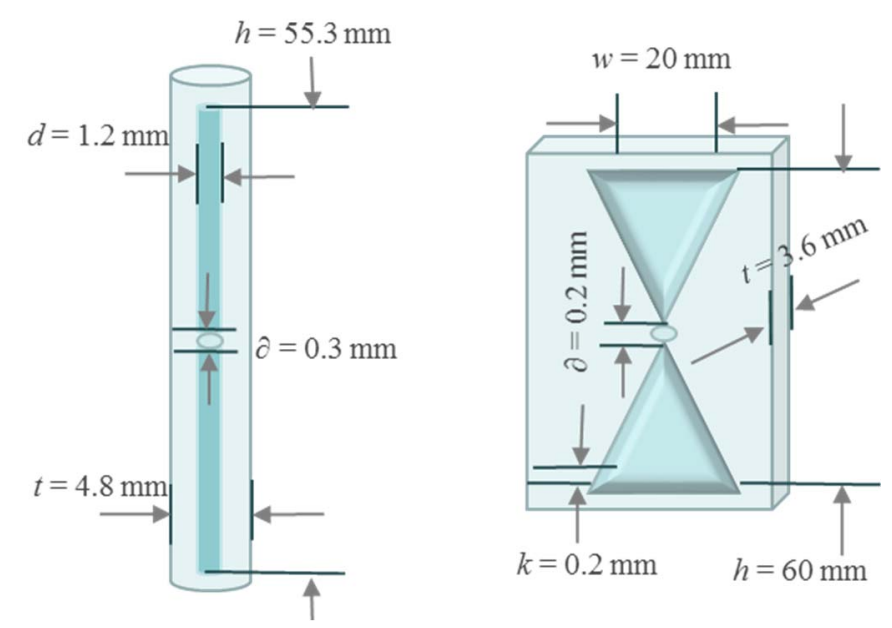

Fig. 1 Insulated cylindrical and bowtie dipole antennas. 




Fig. 2 schematic of the antenna arrangement inside the three-layer phantom for scenario 1(top), scenario 2 (bottom left), scenario 3 (bottom right).

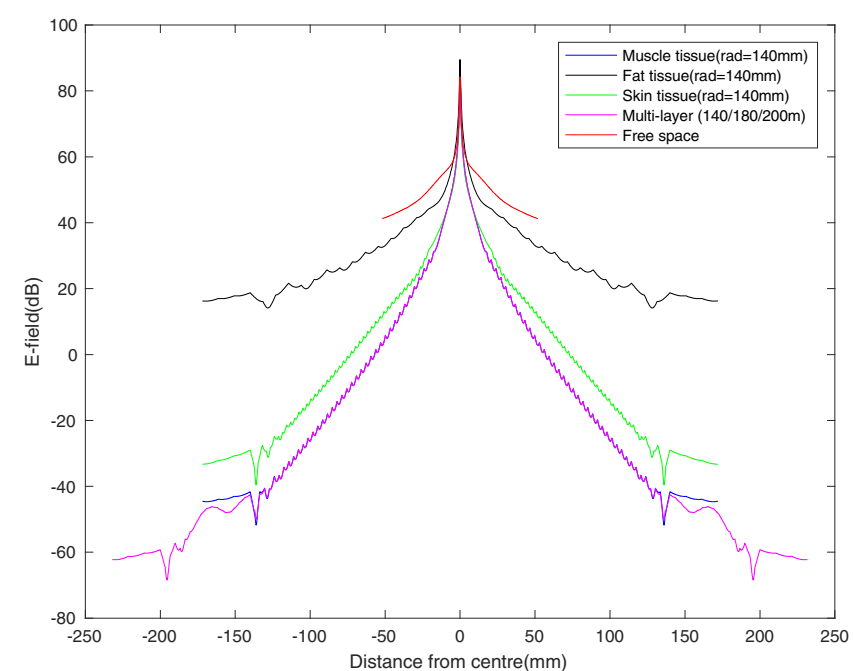

Fig. 3 Total electric field of un-insulated bowtie antenna at $2.38 \mathrm{GHz}$ in freespace, different homogenous phantoms and the multi-layer phantom.

Stage 2: Three different implant to implant scenarios were simulated for the un-insulated bowtie dipole antennas. Fig. 4 shows the forward path gain $\left(\left|S_{21}\right|\right)$ results obtained for Scenario 1 at different antenna separation distances $(3 \mathrm{~mm}$ intervals). The points illustrated in blue, red, black represent the cases where the second antenna is within muscle, fat and skin tissue, respectively. The graph demonstrates how the gain decreases more rapidly in the muscle with almost $3 \mathrm{~dB}$ less gain for every $3 \mathrm{~mm}$ increase in separation. The slope in muscle tissue between $30 \mathrm{~mm}$ and $130 \mathrm{~mm}$ is $-0.75 \mathrm{~dB} / \mathrm{mm}$, indicative of the relatively high attenuation factor at $2.38 \mathrm{GHz}$.

Fig. 5 shows the results of the Scenario 2 simulation where both antennas are at a fixed separation of $90 \mathrm{~mm}$ within the muscle layer but the radius of the fat/muscle tissue boundary is adjusted. The graph illustrates that the gain remains virtually unchanged with the change in the fat layer thickness behind it. This indicates that the communication channel between the antennas is unaffected by the tissue boundary or its properties surrounding the channel.



Fig. 4 Forward gain $\left(\left|S_{21}\right|\right)$ for link between the un-insulated bowtie dipoles in Scenario 1 as the second antenna changes position. The symbol color indicates the tissue type surrounding the second antenna (muscle, fat, skin).

In Scenario 3 the second antenna was positioned within the fat tissue layer but very close to the fat/skin boundary at a radius of $185 \mathrm{~mm}$. In contrast to the Scenario 2 case, the results in Fig. 6 show that as the fat thickness increases by adjustment of the muscle/fat boundary, the forward gain recorded decreases.

Fig. 7 shows a comparison between the Scenario 1 results obtained for insulated and un-insulated (Fig. 4) antennas inside the multi-layered phantom. As expected, the results show a decrease in the losses when the same antenna is insulated. Additionally, the graph also shows the difference between using the bowtie antenna with the broadband characterstics and the dipole antenna when both are insulated.

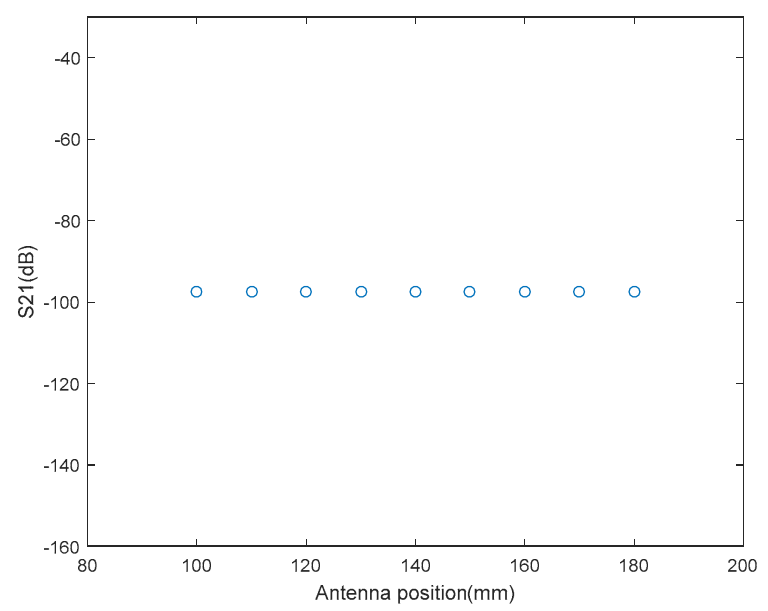

Fig. 5 Forward gain $\left(\left|S_{21}\right|\right)$ for the link between the un-insulated bowtie dipoles in Scenario 2 where they have a fixed $90 \mathrm{~mm}$ separation in the muscle layer and the radius of the fat/muscle boundary is adjusted. 


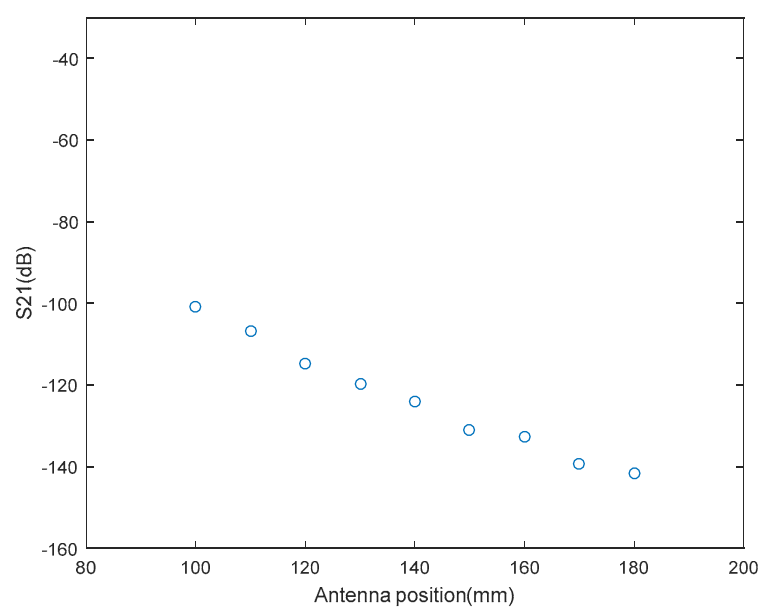

Fig. 6 Forward gain $\left(\left|S_{21}\right|\right)$ for link between un-insulated bowtie dipoles with fixed $185 \mathrm{~mm}$ separation such that the second antenna is in the fat layer and the radius of the fat/muscle boundary is adjusted (Scenario 3 ).

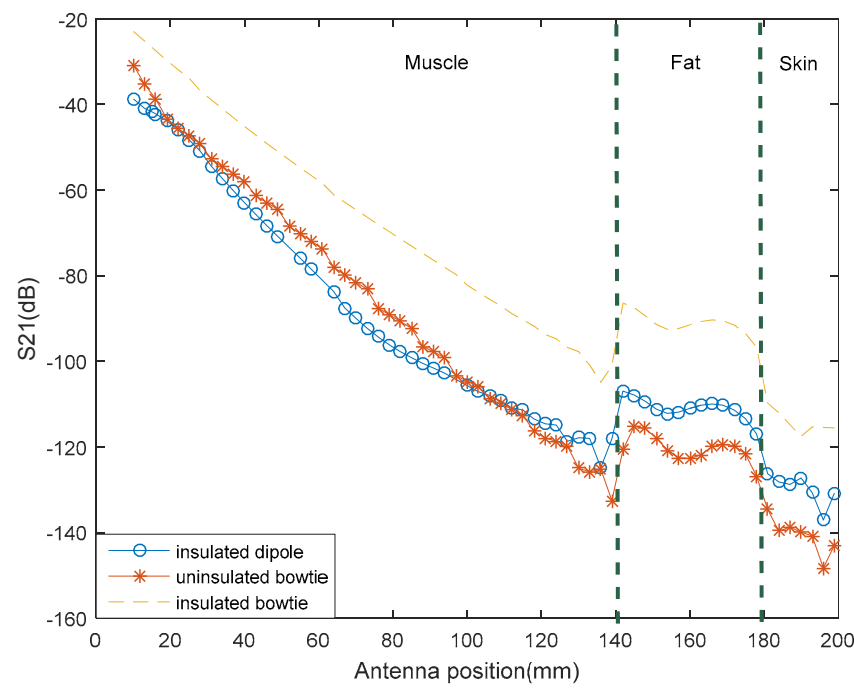

Fig. 7 Comparison of effect of antenna type on the forward gain $\left(\left|S_{21}\right|\right)$ for the link between antennas in Scenario 1 as the second antenna changes position.

\section{DISCUSSION}

The results presented highlight the importance of the propagation channel between implanted antennas in an intrabody network. The electric field distribution from a single implant antenna (Fig. 3) is heavily dependent on the different dielectric properties of the tissue materials. The results confirm the effect of tissue boundaries on the field, as at every boundary (140 $\mathrm{mm}$ and $180 \mathrm{~mm}$ in the multilayer case), there is a significant reduction in the total electric field. When comparing the muscle phantom with the multi-layered phantom, as expected, the electric field behaves the same even until it passes into the next tissue. Additionally, as shown in Fig. 4, at tissue boundaries there is a reduction in the forward path gain between two identical antennas. Moreover, the results also highlight the difference in the gain obtained from the three different tissue types as there is an increase in the gain as the second antenna suffers lower near field losses in the less conductive medium of fat tissue which is also visible with the insulated antennas presented in Fig. 7. In Fig. 5 the path gain is affected by a tissue boundary beyond the direct path between the antennas, even when one of the antennas is only $10 \mathrm{~mm}$ from the boundary.
Fig. 6 illustrates the effect of a variable muscle/fat boundary for two antennas at a fixed distance apart. The decrease in the gain as the position of the boundary increases indicates that the increase in the path length in the more lossy muscle tissue causes more signal attenuation, but the results also show some boundary reflection effects as the pattern is not strictly linear (in $\mathrm{dB}$ terms). The offset in the results in Fig. 7 illustrates how the insulation layer improves the initial near-field tissue losses for both antennas in an IBN link. Moreover, the bowtie antenna behaves better than the dipole when both are insulated mainly due to its larger physical dimensions. However, as expected the results for all antennas follow the same path-loss trend since the attenuation constants remain unchanged in the same phantom.

\section{CONCLUSION}

This numerical study of implant to implant antenna links demonstrates the effect of different tissue boundaries both within and outside the intra-body channel. For the cases considered, the high attenuation rate in muscle tissue at $2.38 \mathrm{GHz}$ ensures that the dominating factor in the IBN channel is the direct path. This is confirmed by the results that show that changes in tissue boundaries beyond this direct path have little effect on the forward path gain. Future work will consider the effect of orientation of the bowtie antennas with respect to each other. The current work was conducted for the MEDradio band but the analysis should be extended to other potential operating frequencies to establish the general principles for IBN links. These studies will also support the development of new antenna concepts for IBN applications. Finally, the work should be experimentally validated in a suitable phantom test bed.

\section{REFERENCES}

[1] D. B. Smith, D. Miniutti, T. A. Lamahewa \& L. W. Hanlen, "Propagation Models for Body-Area Networks: A Survey and New Outlook," IEEE Antennas Propag. Mag., vol. 55, no. 5, pp. 97-117, Oct. 2013.

[2] E. Chow, M. Morris \& P. Irazoqui, "Implantable RF Medical Devices: The Benefits of High-Speed Communication and Much Greater Communication Distances in Biomedical Applications," IEEE Microw. Mag., vol. 14, no. 4, pp. 64-73, Jun. 2013.

[3] A. Khaleghi \& I. Balasingham, "On selecting the frequency for wireless implant communications," Loughborough Antennas \& Propagation Conference (LAPC), pp. 1-4, 2015.

[4] M. K. Magill, G. A. Conway \& W. G. Scanlon, "Robust implantable antenna for in-body communications," Loughborough Antennas \& Propagation Conference (LAPC), pp. 1-12, 2015.

[5] F. Merli, B. Fuchs, J. R. Mosig \& A. K. Skrivervik, "The Effect of Insulating Layers on the Performance of Implanted Antennas," IEEE Trans. Antennas Propagation, vol. 59, no. 1, pp. 21-31, Jan. 2011.

[6] M. Pourhomayoun, M. Fowler \& Z. Zhanpeng Jin, "A novel method for medical implant in-body localization," Annual International Conference of the IEEE Engineering in Medicine and Biology Society, pp. 5757$5760,2012$.

[7] C. Liu, Y.-X. Guo \& S. Xiao, "Capacitively Loaded Circularly Polarized Implantable Patch Antenna for ISM Band Biomedical Applications," IEEE Trans. Antennas Propagation, vol. 62, no. 5, pp. 2407-2417, May 2014.

[8] W. G. Scanlon, J. B. Burns \& N. E. Evans, "Radiowave propagation from a tissue-implanted source at $418 \mathrm{MHz}$ and 916.5 MHz.," IEEE Trans. Biomedical Engineering, vol. 47, no. 4, pp. 527-34, Apr. 2000.

[9] "Medical Device Radiocommunications Service (MedRadio) | Federal Communications Commission," Wireless Telecommunications, 2016. [Online]. Available: https://www.fcc.gov/general/medical-deviceradiocommunications-service-medradio. [Accessed: 07-Aug-2016].

[10] D. Kurup, W. Joseph, G. Vermeen \& L. Martens, "In-body path loss model for homogeneous human muscle, brain, fat and skin," Proc. $4^{\text {th }}$ European Conf. Antennas and Propagation (EuCAP), 2010, pp. 1-4 